titers increased as birth weight and gestational age increased $(p<0.05)$. AntiHBs titers were not different between the healthy term and sick term infants ( $p>0.05)$. Overall seroconversion rate was $\% 97.3$ (preterm $95.6 \%$, term $98.05 \%$ ). In four infants (2 preterm, 2 term) seroconversion were not sustained. Antibody response were not effected from the presumed risk factors such as fresh frozen plasma, IVIG, exchange transfusion, other blood products transfusions. Inflammatory processes such as sepsis, pneumonia, preterm rupture of membranes had no effect on the titers either. As a conclusion preventable levels of antiHBs were achieved in preterm and sick term infants with the schedule routinely used. We concluded that presumed risk factors and transfusion of blood products did not have negative effect on immune response to Hepatitis $B$ vaccine.

\section{REVIEW OF NEONATES BORN IN BELFAST TRUST IDENTIFIED WITH RISK FACTOR(S) FOR TUBERCULOSIS BUT DID NOT RECEIVE BCG VACCINATION}

doi:10.1136/archdischild-2012-302724.1838

'S Mullen, 'S Christie, ${ }^{2} \mathrm{R}$ Carlisle. ' Community Paediatrics; 'Infectious Diseases, Royal Belfast Hospital for Sick Children, Belfast, UK

Aims Data from the Public Health Agency (PHA) for 2009-10 identified 70 infants from the Belfast Trust who had been identified with a risk factor for Tuberculosis (TB) as not receiving the BCG vaccination prior to discharge-theoretically missing $16 \%$ of this population (total with risk factor 438).

Given the rise in TB, with figures in 2009 from the Health Protection Agency (HPA) identifying 9,040 cases in the UK, our aim was to review the data to clarify why these infants were being missed.

Methods We reviewed the neonatal notes for those infants who did not get the BCG vaccination despite a positive risk factor, categorising as per results section. We further reviewed the data for parental refusal to analyse documentation.

Results Unable to access $7 \%$ of charts.

Parental refusal 23\%, received vaccination 17\%, deferred for medical reason $17 \%$, no identifiable risk factor in hospital 12\%, medical decision not required $10 \%$, missed $7 \%$, transferred prior to discharge $4 \%$, transcription error $3 \%$.

Parental refusal group $-50 \%$ clarified the risk factor, $29 \%$ documented risks vs benefits explained, $43 \%$ offered follow-up advice and $35 \%$ documented written info given.

Conclusions $1.1 \%$ were truly missed (5/438).

Parental refusal was the largest category for not receiving vaccination and had poor documentation to explain why. We identified a breakdown in how data is transported to $\mathrm{PHA}$ /or recorded in PHA.

We recommended education for staff, highlighting the need for proper documentation. We also recommended a review of how information was passed to the PHA.

\section{THE ROLE OF CFU OF BCG VACCINE IN TUBERCULIN SKIN REACTION}

doi:10.1136/archdischild-2012-302724.1839

F Fallah, G Eslami, R Pourbaba, H Goudarzi, S Taheri, N Marhamati. Pediatric Clinic Infection Research Center, Tehran, Iran

In view of the importance of tuberculosis in some countries BCG vaccination is highly effective in preventing the development of tuberculosis immediately at birth. The aim of this study is evaluation of BCG by PPD.
Method In this study, the inoculum of live BCG organisms in vaccinated infants at birth was evaluated and the correlation between amount of CFU and PPD reaction were examined at 3 months of age. For this purpose, 854 newborn infants in health centers affiliated to Shaheed Beheshti University of Medical Sciences were studied at the age of 3 months. Tuberculin skin tests were performed in the pasteur Institute and other health centers. After 72 hours, PPD induration diameter was measured. The comparison of the results with chi - square test showed there is a significant correlation between the two variables (size of PPD reaction and live organisms of BCG vaccination).

Results After evaluating live organisms in BCG vaccination, it was concluded that the amount of live organisms in many vaccines is more than 3 milliom while others are less. The following have been obtained as a result of assessment of PPD reaction in vaccinated infants: those vaccinated by live orgrnisms, more than 3 million $93.4 \%$ showed PPD positive reaction in vaccinaged infants but less than 3 million $69.2 \%$ showed PPD positive reaction.

Conclusion Thus, the number of live organisms in vaccination can play an effective role in the size of tuberculin skin reaction.

\section{OUTBREAKS, OUTCRIES, OUTLIERS: WHO RESPONDS TO MEASLES ELIMINATION GOALS IN THE EUROPEAN REGION WITH INNOVATIVE BEHAVIOUR CHANGE TOOLS}

doi:10.1136/archdischild-2012-302724.1840

${ }^{1} \mathrm{R}$ Butler, ${ }^{2} \mathrm{~N}$ Likhite. ${ }^{1}$ Vaccine Preventable Diseases and Immunization; ${ }^{2}$ Consultant with Vaccine Preventable Diseases and Immunization, WHO Regional Office for Europe, Copenhagen, Denmark

Background and Aims Measles outbreaks in the World Health Organisation (WHO)'s European Region continue to threaten the achievement of the 2015 elimination goal. Over 37,000 measles cases were reported in 2011 alone. Failure to vaccinate is the principal obstacle to optimal national and sub-national immunity. In a context where loss of public confidence in vaccination is often cited, experts deplore the presence of vaccination outliers and outcries of anti-vaccine lobbyists.

Generating demand for vaccination is a pivotal strategy for elimination. However, the traditional "one-size fits all" approach to immunisation communications no longer meets current needs. WHO's aim is to offer a toolkit for Member States to design effective targeted vaccination demand generation campaigns.

Methods A review of literature on vaccination behaviours shows that reasons for not vaccinating are multiple and complex. Drawing on international best practices in health behaviour change communications and social marketing, WHO Regional Office for Europe has developed an adaptable, innovative conceptual framework and tools to help countries

1. understand and identify environmental opportunity, supportive ability and personal motivation determinants of vaccination behaviours,

2. target susceptible populations and

3. tailor evidence-based programmes to increase uptake of vaccination

Results and conclusions Building Member States' capacity to profile and tailor responses to susceptible populations will result in higher vaccination coverage and accelerate progress towards eliminating measles in the European Region. The approach and tools will be piloted in two Member States in 2012. Further, they will be implemented by WHO in partnership with Ministries of Health from 2013 on. 